Published in final edited form as:

J Matern Fetal Neonatal Med. 2016 March ; 29(5): 803-806. doi:10.3109/14767058.2015.1019457.

\title{
First trimester fetal heart rate as a predictor of newborn sex
}

\author{
L. A. Bracero ${ }^{1}$, D. J. Seybold², S. Witsberger², L. Rincon'², A. Modak², and L. V. Baxi ${ }^{3}$ \\ ${ }^{1}$ Department of Obstetrics and Gynecology, West Virginia University, Charleston Campus, \\ Charleston, WV, USA \\ ${ }^{2}$ Center for Health Services \& Outcomes Research, Charleston Area Medical Center Health \\ Education and Research Institute, Charleston, WV, USA \\ ${ }^{3}$ Department of Obstetrics and Gynecology, New York University Langone Medical Center and \\ School of Medicine, New York, NY, USA
}

\begin{abstract}
Objective-To predict the sex of newborns using first trimester fetal heart rate (FHR).

Methods-This was a retrospective review of medical records and ultrasounds performed between 8 and 13 weeks of gestation. Continuous variables were compared using Student's $t$-tests while categorical variables were compared using Chi-square test.
\end{abstract}

Results-We found no significant differences between 332 (50.7\%) female and 323 (49.3\%) male FHRs during the first trimester. The mean FHR for female fetuses was $167.0 \pm 9.1 \mathrm{bpm}$ and for male fetuses $167.3 \pm 10.1 \mathrm{bpm}(p=0.62)$. There was no significant difference in crown rump length between female and male fetuses $(4.01 \pm 1.7$ versus $3.98 \pm 1.7 \mathrm{~cm} ; p=0.78)$ or in gestational age at birth $(38.01 \pm 2.1$ versus $38.08 \pm 2.1$ weeks; $p=0.67)$. The males were significantly heavier than females ( $3305.3 \pm 568.3$ versus $3127.5 \pm 579.8 \mathrm{~g} ; p<50.0001)$ but there were no differences in the proportion of small for gestational age (SGA), average for gestational age (AGA) and large for gestational age (LGA) infants.

Conclusions-We found no significant difference between the female and male FHR during the first trimester in contrast to the prevailing lay view of females having a faster FHR. The only statistically significant difference was that males weighed more than female newborns.

\section{Keywords}

Fetal heart rate; fetal sex; first trimester; newborns; ultrasound

\footnotetext{
*Presented at the 22nd World Congress on Ultrasound in Obstetrics and Gynecology, 9-12 September 2012, Copenhagen, Denmark. Address for correspondence: Luis A. Bracero, MD, Southside Hospital, 301 East Main Street, Bay Shore, NY 11706, USA. Tel: +1 631968 3795. Fax: +1 631864 5288. Lbracero@NSHS.edu.

Declaration of interest

The authors report no declaration of interest.
} 


\section{Introduction}

Expectant parents are often curious and request fetal sex identification. Ultrasound (US) during the second and third trimesters can be reliably used to determine the sex of the fetus. The accuracy of US before 14 weeks gestation in identifying the sex of the fetus is poor [1]. There is a prevailing belief in the general population that the female fetus has a more rapid heart rate than the male fetus. We found one website that encourages prospective parents to use fetal heart rate (FHR) to predict the sex of newborns [2]. The purpose of this study was to address this issue during the first trimester of pregnancy when US is often inaccurate in identifying the fetal sex. Our null hypothesis was that there is no significant difference in FHRs between female and male fetuses during the first trimester.

\section{Methods}

This is a study of births at Women and Children's Hospital from January 1, 2006 to April 9, 2010 in which USs were performed between 8 and 13 weeks of gestation at the hospital's Perinatal Diagnostic Center. The medical records and USs of these patients were retrospectively reviewed. The inclusion criteria were: singleton pregnancies with documented FHR that delivered at our hospital and had pregnancy dating by a last menstrual period (LMP) and a confirmatory crown rump length (CRL) measurement or had an uncertain LMP and were dated by the initial CRL. The exclusion criteria were: maternal bradycardia or tachycardia, maternal thyroid disease, maternal cardiac arrhythmias, maternal use of beta blocker agents, maternal anxiety disorder, maternal fever, spontaneous abortion, stillbirths, ambiguous genitalia at birth, fetal malformations, fetal cardiac arrhythmias and fetal chromosomal abnormalities. The Institutional Review Board of the Charleston Area Medical Center approved the study.

US examinations were performed using Acuson Sequoia (Mountain View, CA), Aloka Alpha 10 (Tokyo, Japan) and General Electric Voluson E8 (Rancho Cordova, CA) machines. The Acuson Sequoia machine was equipped with 4.0-8.0 MHz abdominal and vaginal transducers. The Aloka Alpha 10 machine was equipped with 3.0-7.5 MHz abdominal and 3.5-7.5 MHz vaginal transducers. The General Electric Voluson E8 machine was equipped with $2.0-5.0 \mathrm{MHz}$ abdominal and $4.0-9.0 \mathrm{MHz}$ vaginal transducers. The fetal heart rate was obtained using spectral Doppler ultrasound for $4-5$ heart beats while keeping the thermal index for soft tissues and bone as low as possible $(<1)$ in accordance with the As Low As Reasonably Achievable (ALARA) principle. The sex was established at the time of birth.

Descriptive statistics and univariate analyses were used as appropriate for continuous or categorical variables. Continuous variables were presented as means and standard deviations and were compared by using Student's independent samples $t$-tests. Categorical variables were reported as percentages and were compared using Chi-square or Fisher's exact test. An alpha of less than 0.05 was used as a determinant of statistical significance.

We calculated a sample size based on the data from McKenna et al. [3]. They found that during the first trimester, male fetuses had a slightly higher average FHR when compared to 
female fetuses $(154.9 \pm 22.8 \mathrm{bpm}$ versus $151.7 \pm 22.7 \mathrm{bpm} ; p=0.13)$. We determined that the enrollment for the current study should be 1600 pregnant women with a goal of including 794 males and 794 females based on a FHR difference of 3.2 beats per minute (bpm) between the male and female fetuses and an alpha level of 0.05 with $80 \%$ power. We planned to have an over enrollment to provide adequate numbers for each gender since the delivery rate for females is slightly lower at $49.5 \%$ than for males. Statistical analysis was done using SAS 9.3 (SAS Institute, Cary, NC).

\section{Results}

An interim analysis was performed after data on 655 patients were collected. The study was stopped because there was a very small absolute difference between the sexes $(0.3 \mathrm{bpm})$. We calculated that it would require a sample size of over 12000 participants to detect a significant difference in FHR and this led us to believe that there would be no statistically significant difference between the FHR of female and male fetuses.

Our study population consisted of 655 mothers and their fetuses. The mean maternal age was $24.2 \pm 6.0$ years, ethnicity was primarily white $(91.2 \%)$ and the majority were multiparous (57.3\%). Additional maternal characteristics and birth outcome comparisons between male and female newborns are shown in Table 1 . The primary US indication was for an uncertain or unknown LMP (37.7\%). We found no significant differences between $332(50.7 \%)$ female and $323(49.3 \%)$ male FHRs during the first trimester. The mean FHR for female fetuses was $167.0 \pm 9.0 \mathrm{bpm}$ and for male fetuses $167.3 \pm 10.1 \mathrm{bpm}(p=0.62)$. Likewise, a breakdown of FHR by 8, 9, 10,11, 12 and 13 weeks showed no difference in FHR between the sexes (Figure 1). The CRL was also not significantly different between the female and male fetuses $(4.01 \pm 1.7 \mathrm{~cm}$ versus $3.97 \pm 1.6 \mathrm{~cm} ; p=0.78)$ and neither was gestational age at birth $(38.0 \pm 2.1$ weeks versus $38.1 \pm 2.1$ weeks; $p=0.67)$. The male newborns were significantly heavier than the females $(3305.3 \pm 568.3 \mathrm{~g}$ versus $3127.5 \pm$ $579.8 \mathrm{~g} ; p<0.001$ ) with no differences in the proportion of small for gestational age (SGA), average for gestational age (AGA) and large for gestational age (LGA) infants. The FHR for male versus female fetuses that were born SGA $(163.9 \pm 8.1 \mathrm{bpm}, n=14$ versus $164.1 \pm$ $12.0 \mathrm{bpm}, n=14 ; p=0.4411)$, AGA ( $167.5 \pm 10.5 \mathrm{bpm}, n=232$ versus $166.7 \pm 9.3 \mathrm{bpm}, n$ $=229 ; p=0.3434)$, LGA (162.4 $\pm 12.5 \mathrm{bpm}, n=5$ versus $168.7 \pm 11.6 \mathrm{bpm}, n=3 ; p=$ $0.5088)$ and preterm $(166.1 \pm 10.9 \mathrm{bpm}, n=48$ versus $168.8 \pm 9.5 \mathrm{bpm}, n=51 ; p=0.1813)$ was not statistically significantly different during the first trimester. The rate of preterm births ( $<37$ weeks gestation) was similar for both male and female sexes; $14.9 \%$ for males and $15.7 \%$ for female newborns $(p=0.78$ ). The FHRs for male and female fetuses that were subsequently admitted to the Neonatal Intensive Care Unit (NICU) were also not statistically significantly different during the first trimester $(165.0 \pm 8.6 \mathrm{bpm}, n=18$ versus $167.1 \pm 8.6$ bpm, $n=24 ; p=0.4411)$.

\section{Discussion}

The topic of FHR and gender prediction has been studied mostly during the third trimester of pregnancy. Many of these studies have involved small sample sizes and the results have been mixed. Some studies were done during the antepartum period, others during early labor 
and still others during late labor. Bernard [4] studied 45 female and 50 male fetuses weekly during the last 8 weeks of pregnancy and found no differences in the mean FHR between the sexes. Hall et al. [5] assigned sex to 100 fetuses at 32 weeks gestation using averaged FHR recordings from previous prenatal visits. Female sex was assigned if the FHR equaled or exceeded $140 \mathrm{bpm}$ and male sex was assigned if the FHR was less than $140 \mathrm{bpm}$. They found the FHR to be a good forecaster of the newborn's sex, reporting a positive predictive value of $91 \%$ for males and $74 \%$ for females. Ostler and Sun [6] reported on a convenient sample of 20 pregnancies that had a single FHR obtained with a Doppler fetoscope after the 26th week of gestation. The women gave birth to 10 male and 10 female infants. They used the $140 \mathrm{bpm}$ FHR cut-off to predict the sex of the newborn. They report a sensitivity of $10 \%$ and specificity of $60 \%$ for predicting males and a sensitivity of $60 \%$ and specificity of $10 \%$ for predicting females. Petrie and Segalowitz [7] studied 250 female and 250 male fetuses during early labor and found that the mean difference in the FHR between them was not statistically significant. Dawes et al. [8] analyzed the FHR records of 1884 term pregnancies, 890 female and 994 male fetuses, during the last hour prior to delivery. They found that female fetuses spent more time with FHR $>150 \mathrm{bpm}$ while male fetuses spent more time with FHR <120 bpm $(p<0.0001)$. Arguably, other variables affect the FHR during labor.

Druzin et al. [9] performed non-stress tests on 12 female fetuses and 25 male fetuses between 19 and 40 weeks of gestation. They reported no differences in baseline FHR between the female and male fetuses. Genuis et al. [10] followed 212 singleton pregnancies between 14 and 41 weeks gestation with antepartum FHR testing. They computed the overall FHR averages for each pregnancy and found no significant difference between the antenatal FHRs of male and female fetuses during the second and third trimesters of pregnancy. There is one abstract publication comparing male and female FHRs during the second trimester. Neiger et al. [11] reported on the FHR of 57 male and 43 female fetuses between 18 and 23 weeks of gestation. The fetal sex was identified by ultrasound examination. There was no significant difference in the average FHR between the male and female fetuses.

McKenna et al. [3] analyzed the FHR obtained at less than 14 weeks gestation according to the sex of the fetus. They found no significant differences between the 244 female and 233 male FHRs studied. The average FHRs in our study for both male and female fetuses were higher and the standard deviations were lower than those they reported. The difference between our first trimester FHRs for male infants $(167.3 \pm 10.1, n=323$ versus $154.9 \pm$ 22.8, $n=233 ; p=0.0001)$ and for female infants $(167.0 \pm 9.1, n=332$ versus $151.7 \pm 22.7$, $n=244 ; p=0.0001$ ) was statistically significantly higher when compared to the results reported by McKenna and colleagues. They included patients below 14 weeks gestation but did not provide the actual gestational age ranges. It is possible that they included pregnancies at 6-7 weeks gestation when the FHRs are known to be lower when compared to pregnancies between 8 and 14 weeks of gestation [12].

Male newborn infants at term on average are known to weigh $150 \mathrm{~g}$ more than female newborns [13]. We found that our male newborns weighed $178 \mathrm{~g}$ more than female newborns. Leicester [14] while working in India reported on the FHR of 550 term or near 
term uncomplicated pregnancies before labor or during the early stages of labor. He divided the population into Europeans, Natives of India and East Indians (mixed European and Native). He found no significant difference between the average FHR of male and female newborns. He also looked at the influence of fetal weight on FHR, since the FHR differences between the sexes have been based on the assumption that the FHR is inversely related to the fetal weight. In the Native group the average FHR diminished as the weight of the newborn increased. However, he did not find a similar relationship in the European and East Indian groups. He reported that the fetal weight has some influence on the rate of the fetal heart. Coughlin [15] reviewed the medical charts of 233 women who gave birth to 123 male and 110 female infants. The average FHRs between the sexes was not significantly different. Like us, she found that males weighed more than female newborns ( $3428 \mathrm{~g}$ versus $3221 \mathrm{~g}, p=0.001$ ) but there was no significant relationship between third trimester FHR and newborn weight. Bernard [4] also found no relationship between the average FHR during the last 8 weeks of pregnancy and the size of the newborns. In our population, there was no significant difference in the size of the fetuses as determined by the fetal CRL measurements during the first trimester when our FHR measurements were obtained.

The strength of our study is that it excluded patients with maternal and fetal conditions that could have influenced the FHR. We included only FHRs between 8 and 13 weeks gestation when the FHR is more uniform. We analyzed the FHR by the distinct gestational ages as well as in aggregate. We also analyzed the FHR based on outcomes, such as preterm births, NICU admissions, SGA, AGA and LGA infants.

In conclusion, we found no significant differences between female and male FHR during the first trimester. Therefore, expectant parents should not rely on first trimester FHR to determine the sex of their baby.

\section{References}

1. Odeh M, Granin V, Kais M, et al. Sonographic fetal sex determination. Obstet Gynecol Surv. 2009; 64:50-7. [PubMed: 19099612]

2. [1 Oct 2014] Pink? Blue? You're pregnant.. Now you want to know the sex of your baby. Available from: http://www.babybpm.com/

3. McKenna DS, Ventolini G, Neiger R, Downing C. Gender-related differences in fetal heart rate during the first trimester. Fetal Diagn Ther. 2006; 21:144-7. [PubMed: 16354993]

4. Bernard J. Prediction from human fetal measures. Child Dev. 1964; 35:1243-8. [PubMed: 14251370]

5. Hall S, King K, Cox SM. Fetal sex prediction: the heart of the matter. J KY Med Assoc. 1993; 91:409-10. [PubMed: 8228583]

6. Ostler S, Sun A. Fetal sex determination: the predictive value of 3 common myths. CMAJ. 1999; 161:1525-6. [PubMed: 10624410]

7. Petrie B, Segalowitz SJ. Use of fetal heart rate, other perinatal and maternal factors as predictors of sex. Percept Mot Skills. 1980; 50:871-4. [PubMed: 7402870]

8. Dawes NW, Dawes GS, Moulden M, Redman CW. Fetal heart rate patterns in term labor vary with sex, epidural analgesia, and fetal weight. Am J Obstet Gynecol. 1999; 180:181-7. [PubMed: 9914601]

9. Druzin ML, Hutson JM, Edersheim TG. Relationship of baseline heart rate to gestational age and fetal sex. Am J Obstet Gynecol. 1986; 154:1102-3. [PubMed: 3706438] 
10. Genuis S, Genuis SK, Ghang W. Antenatal fetal heart rate and maternal intuition as predictors of fetal sex. J Reprod Med. 1996; 41:447-9. [PubMed: 8799924]

11. Neiger R, Ventolini G, McKenna DS, et al. The myth of sex-dependent difference in fetal heart rate. Obstet Gynecol. 2004; 103:31S.

12. Hanprasertpong T, Phupong V. First trimester embryonic/fetal heart rate in normal pregnant women. Arch Gynecol Obstet. 2006; 274:257-60. [PubMed: 16715287]

13. Zhang J, Bowes WA. Birth-weight-for-gestational-age patterns by race, sex, and parity in the United States population. Obstet Gynecol. 1995; 86:200-8. [PubMed: 7617350]

14. Leicester JC. The relation of the frequency of the fetal heart beat to the sex and weight of the child. J Obstet Gynaecol Br Emp. 1907; 12:39-41.

15. Coughlin LL. Do fetal heart rates predict gender? J Obstet Gynecol Neonatal Nurs. 1993; 22:105. 


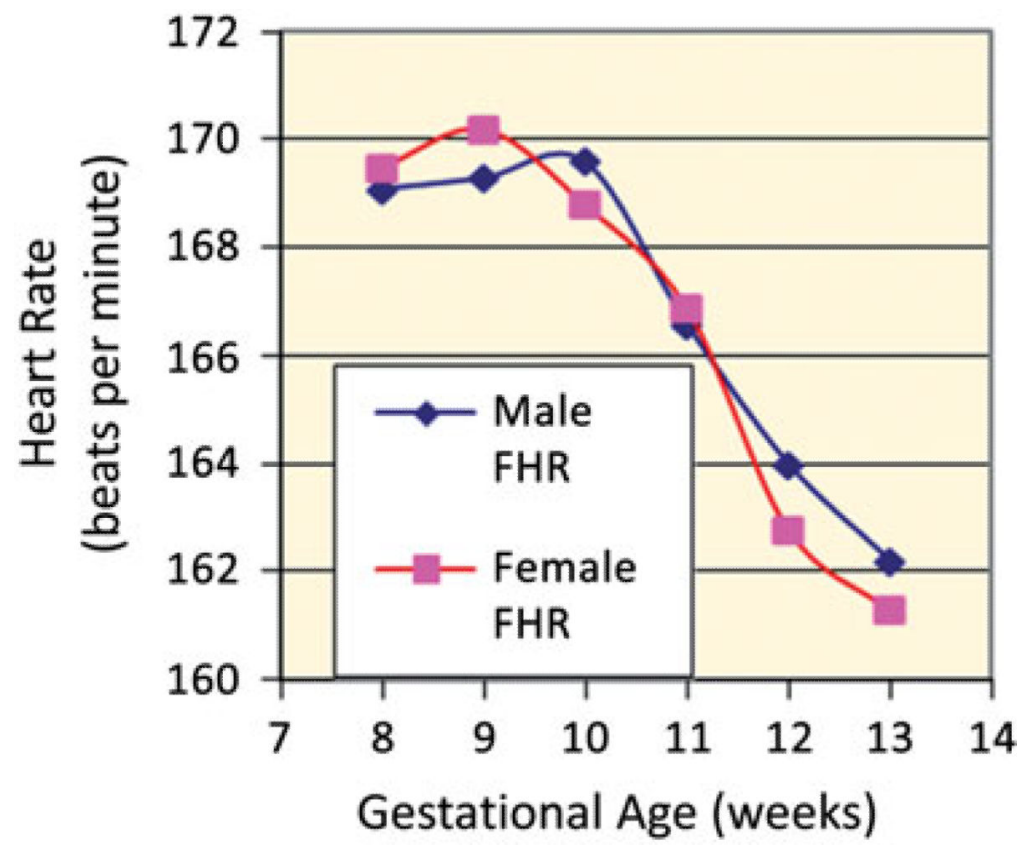

Figure 1.

Male and female heart rate by gestational age. 


\section{Table 1}

Pregnancy characteristics and birth outcomes of male and female newborns.

\begin{tabular}{|c|c|c|c|}
\hline & Male $(n=323) N(\%)$ or mean \pm SD $($ range $)$ & Female $(n=332) N(\%)$ or mean \pm SD (range) & $p$ value \\
\hline Maternal age at delivery & $24.6 \pm 6.1(13-43)$ & $23.7 \pm 6.0(11-47)$ & 0.0884 \\
\hline Ethnicity/race & & & 0.4572 \\
\hline White & $297(91.95 \%)$ & $300(90.36 \%)$ & \\
\hline Black/African American & $25(7.74 \%)$ & $28(8.43 \%)$ & \\
\hline Asian American & $1(0.31 \%)$ & $4(1.20 \%)$ & \\
\hline Nulliparity & $134(41.49 \%)$ & $146(43.98 \%)$ & 0.5284 \\
\hline Ultrasound indication & & & 0.0817 \\
\hline Uncertain/unknown LMP & $123(38.08 \%)$ & $124(37.35 \%)$ & \\
\hline Abdominal pain/cramping & $57(17.65 \%)$ & $48(14.46 \%)$ & \\
\hline Vaginal bleeding & $23(7.12 \%)$ & $47(14.16 \%)$ & \\
\hline Maternal illness (DM, HTN) & $31(9.60 \%)$ & $41(12.35 \%)$ & \\
\hline Nuchal translucency & $32(9.91 \%)$ & $28(8.43 \%)$ & \\
\hline Fetal viability & $24(7.43 \%)$ & $21(6.33 \%)$ & \\
\hline Large or small for dates & $27(8.36 \%)$ & $18(5.42 \%)$ & \\
\hline Nausea/vomiting & $6(1.86 \%)$ & $5(1.51 \%)$ & \\
\hline Crown rump length & $3.97 \pm 1.6$ & $4.01 \pm 1.7$ & 0.7835 \\
\hline Gestational age at birth (weeks) & $38.1 \pm 2.1(24-42)$ & $38.0 \pm 2.1(28-41)$ & 0.6688 \\
\hline Weight of fetus (g) & $3305.3 \pm 568.3(660-4935)$ & $3127.5 \pm 579.8(940-4770)$ & $<0.001$ \\
\hline
\end{tabular}

